\title{
Out-patient referrals of major depression to psychiatrists in central Liverpool
}

\author{
Ben GreEn, Registrar to the Academic Team, Royal Liverpool Hospital, Liverpool \\ L63 3BX (correspondence), and M. AMIN EL-HIHI, Senior House Officer, \\ Rainhill Hospital, Merseyside
}

Psychiatric clinics see $4 \%$ of all hospital referrals by general practitioners (GPs), but the literature contains surprisingly little about the referral behaviour of, and the patients referred by, GPs to psychiatrists (Wilkinson, 1989). We describe an analysis of the Royal Liverpool Hospital psychiatric out-patient clinic which serves a deprived inner city population of 125,000 people and its GPs.

\section{The study}

For this retrospective, descriptive study we looked at the out-patient records of all new patients referred to the clinic by GPs from 1 January 1986 to 31 December 1988. These patients had had no past psychiatric contact and so we were considering only first episodes of psychiatric illness.

From each patient's record we took the month and year of referral, sex, age, marital status, occupation, number of children, social situation, length of illness before seeing a psychiatrist, GP assessment or diagnosis and GP treatment, consultant diagnosis, disposal, treatment and time elapsing before declared well.

We made an artificial division into two groups to perform a statistical analysis. One group contained only cases of major depression (classified according to the diagnostic criteria for a major depressive episode in DSM-III-R). The second 'non-depressed' group contained all other diagnoses.

\section{Main findings}

During the three years GPs referred 254 new patients. Sixty-one $(23.9 \%)$ fulfilled the criteria for a major depressive episode ( 22 males and 39 females). The non-depressed group contained 81 males and 112 females. The mean age of patients in the depressed group was 38.4 years (s.d. $=13.2)$ and in the non-depressed group 33.64 years $(s . d .=13.32)$. The most frequent diagnoses in the non-depressed group were anxiety or adjustment disorder (77 cases), followed by personality disorder ( 40 cases).

The most frequent occupation in the depressed group was that of housewife ( 24 or $34 \%$ ) followed in size by unemployment ( 12 or $20 \%$ ). The unemployment rate for central Liverpool at this time was $30.65 \%$. Indeed, the group with major depression had a lower unemployment figure of $19.67 \%$ and the non-depressed group had a higher figure $(38.3 \%)$. A $\chi^{2}$ test of employment v. unemployment in the depressed v. non-depressed groups yielded a score of 6.2 (one degree of freedom, associated probability of, or less than, 0.012). This would suggest a significant difference between the two diagnostic groups as far as this risk factor is concerned, and might suggest that major depression is more biologically determined while anxiety and adjustment disorders (which made up the bulk of the non-depressed group) are more linked to adverse circumstances.

\section{GP referral letters}

When we looked at the GP referral letters for the patients in the depressed group, $65 \%$ of the letters gave the diagnosis as some form of depression. Twenty-three per cent described the diagnosis in terms of anxiety, neurosis or nervous distress. The remaining $12 \%$ were equally distributed between the diagnoses of agoraphobia, alcoholism, schizophrenia and manic-depressive psychosis. The most common stated reason for the GP referral was the 'severity of the depression' ( $48 \%$ of letters). Twenty per cent of referrals gave resistance to treatment as the reason for referring the patient and $15 \%$ were referred because of the chronicity of their illness. Suicide attempts prompted referral in $9 \%$.

In the non-depressed group there were 49 cases (about 25\%) where the diagnoses of psychiatrists and GP matched. Ninety-three patients had been given the diagnosis of depressive illness by their GP but did not conform to the psychiatric notion of a major depressive illness. In some of these cases the GPs were using the notion of a depressive neurosis which is not to be found in DSM-III-R. These were re-classified by the psychiatrist. Fifty-seven were given DSM-III-R diagnoses of an anxiety disorder or an adjustment with affective features, and a further 33 had a personality disorder. Another three were alcoholics. 


\section{Treatment}

Only three cases of depression needed in-patient care. Six $(10 \%)$ went to the day hospital and three $(5 \%)$ were managed at home by the GP. Two were managed using community visits by the consultant. The majority of $47(77 \%)$, was treated in out-patients.

The non-depressed group was managed differently, $74(38 \%)$ were managed in out-patients, but another $60(31 \%)$ were returned to the care of their own general practitioner. Twenty-one $(11 \%)$ were admitted to the day hospital. Eighteen were admitted $(9 \%)$ usually suffering with schizophrenia. Twenty were referred to the Department of Psychotherapy $(10 \%)$.

Twenty-seven (44\%) of the depressed group received antidepressants from their GP before seeing the psychiatrist. The GPs' most popular choice for these cases was dothiepin, used in 16. The mean dose of tricyclics prescribed by GPs was $77.8 \mathrm{mg}$. Seventeen depressed patients received only benzodiazepines from their GP.

In 1986 GPs had made 20 benzodiazepine prescriptions for the referred patients (nine in the depressed group), but in 1988 this had fallen to four (with only two being in the depressed group). We used a $\chi^{2}$ test to assess any real change in the number of benzodiazepine prescriptions over the period of the study. This gave a $\chi^{2}$ score of 17.03 (two degrees of freedom so that $P$ was less than or equal to 0.0003 ). We concluded that benzodiazepine prescribing had significantly reduced over three years.

Psychiatrists prescribed antidepressant medication in virtually every case of depression, and concentrated antidepressants almost solely within this group. The overwhelming choice was some form of tricyclic, again usually dothiepin (32 prescriptions). Psychiatrists used a higher dose than GPs (mean tricyclic dosage $=112.9 \mathrm{mg}$ ).

\section{Outcome of the illness}

The mean duration of the depressive illness before seeing the psychiatrist was about 13 months. After seeing the psychiatrist and a change in treatment the interval until well was about 3.5 months. In only one patient was there no improvement with treatment.

A large number of patients in the non-depressed group defaulted from clinic follow-up (85 patients or $44 \%$ ) or were returned to GP care (45 patients or $23 \%$ ). This high proportion of patients not followedup markedly distinguishes the depressed group from the non-depressed group.

\section{Comment}

Previous studies of diagnoses in out-patient clinics in Manchester and Plymouth give rates of $34 \%$ and $42 \%$ respectively for depression (Johnson, 1973 and
Kessel \& Hassall, 1965). Clearly the criteria that are chosen to make up a 'case' of depression are crucial. Johnson, for instance, used the Glossary of Mental Disorders. The DSM-III-R criteria for a Major Depressive Episode tend to put emphasis on objective biological features and hence restrict our figure to $23.9 \%$.

GPs diagnosed depression only in $65 \%$ of cases later diagnosed as major depression, and overall the agreement between the two professional groups might partially be explained by the fact that GPs have been said to have different criteria for establishing what is a 'case' (Goldberg, 1982).

In spite of the marked reduction in benzodiazepine prescriptions during the three years of the study, there was no compensatory increase found in other psychotropic medication or vaunted substitutes for benzodiazepines such as propranolol. Adverse publicity in the lay and medical press may have persuaded GPs not to prescribe benzodiazepines in new cases, or alternatively persuaded their patients to refuse or not to request such prescriptions. The reduction in prescriptions in 1988, without a compensatory rise in other psychotropic drugs, might now indicate a new general reluctance to prescribe anything to psychiatric cases. The exact significance of this new fashion in prescribing has not yet emerged.

The new patient referral rate of the city centre GPs was only 6.77 per 10,000 per year. Among hospitals in East Anglia the referral rate for psychiatric outpatient clinics is 25 per 10,000 per year (Moore \& Roland, 1989). The discrepancy might be accounted for by the suggestion that GPs in central Liverpool are more likely to refer patients directly to the accident and emergency department. This bias is added to by self-referrals to the Royal Liverpool casualty (among the busiest in Britain), and requests for consultant domiciliary visits. Almost three times as many new cases were referred to psychiatric care via these alternative routes. We are aware that a referral bias must exist between these different avenues to a psychiatric consultation.

Many of the best studies looking at GP referrals to psychiatric out-patient departments are over 15 years old. Changes have undoubtedly occurred in the classification and treatment of psychiatric disorders and in the development of psychiatric services for the community. These changes have important consequences and yet they have been only sporadically documented in research. It may be that improved information technology and medical audit will generate an enhanced picture of the national interface between primary and secondary care. A detailed model of this interface even at the crude level of this paper does not currently exist generally at a Regional level. A national picture will be necessary for developing the theories in Working for Patients. 


\section{Acknowledgements}

We thank M. E. Dewey of the Liverpool University Department of Psychiatry for his statistical advice.

\section{References}

GOLDBERG, D. (1982) The concept of a 'case' in general practice. Social Psychiatry, 17, 61-65.
JoHnson, D. A. W. (1973) An analysis of outpatient services. British Journal of Psychiatry, 122, 301-306.

Kessel, W. I. N. \& Hassall, C. (1965) Psychiatric outpatients in Plymouth - an area service analysed. British Journal of Psychiatry, 111, 10.

MoORE, A. T. \& Roland, M. O. (1989) How much variation in referral rates among general practitioners is due to chance? British Medical Journal, 298, 500-502.

WILKINSON, G. (1989) Referrals from general practitioners to psychiatrists and paramedical mental health professionals. British Journal of Psychiatry, 154, 72-76.

\title{
Writing to the patient
}

\section{Comments of patients who received a letter following an initial psychiatric out-patient consultation}

\author{
John S. Price, Consultant Psychiatrist, Milton Keynes General Hospital, \\ Milton Keynes MK6 5LD; and RaCHEL AsCH, Teaching Fellow, Applied \\ Psychology Unit, College of Aeronautics, Cranfield Institute of Technology, \\ Cranfield, Bedfordshire
}

In a recent study we compared writing to the patient with writing to the general practitioner after an initial psychiatric out-patient consultation. One of us (R.A.) interviewed the patients at home about two weeks after the consultation, and compared the two groups on satisfaction with the consultation, comprehension of information given by the psychiatrist, and compliance with advice. We felt that the comments of the patients might be of interest, particularly to those who might have considered (or done) something similar.

\section{The study}

Patients were recruited for the project from 1 July 1988 to 30 June 1989 . They were referrals by GPs to a psychiatric out-patient clinic in a District General Hospital, manned by a consultant and variably one or two SHOs/registrars (some of whom were on psychiatric and some on general practice rotations).

Patients were seen by the registrar for an hour, then presented to the consultant (usually with the patient present) for about 15 minutes, following which the consultant interviewed the patient for about 15 minutes. A few patients were seen only by the consultant. Neither registrar nor consultant knew until the end of the consultation whether the patient was in the experimental or control group, and it was at that stage that the patient was told about the research. The registrars were instructed to summarise the consultation in their letters to the patient; no format or headings were used; the letters were not checked by the consultant before dispatch.

Patients were interviewed by the research worker at home, and it was pointed out that the interviewer was independent and not connected to the hospital or the psychiatric service. In the section of the interview devoted to the letter, the patients were asked two open-ended questions, "How did you feel about getting the letter?" and, "Did you find the letter useful?" Their replies were recorded, and then they were asked to rate their feelings about the letter and its usefulness on five-point scales.

\section{Findings}

Appointments were sent to 168 patients. Ten rang to cancel or postpone. Thirty-seven did not attend. Of 